\title{
Detection of an immature dentate gyrus feature in human schizophrenia/bipolar patients
}

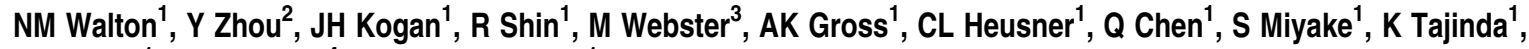 \\ $\mathrm{K}_{\text {Tamura }}{ }^{1}, \mathrm{~T}_{\text {Miyakawa }}{ }^{4}$ and $\mathrm{M}$ Matsumoto ${ }^{1}$
}

Hippocampus-associated cognitive impairments are a common, highly conserved symptom of both schizophrenia (SCZ) and bipolar disorder (BPD). Although the hippocampus is likely an impacted region in SCZ/BPD patients, the molecular and cellular underpinnings of these impairments are difficult to identify. An emerging class of mouse models for these psychiatric diseases display similar cognitive impairments to those observed in human patients. The hippocampi of these mice possess a conserved pathophysiological alteration; we term the 'immature dentate gyrus' (iDG), characterized by increased numbers of calretininpositive immature neuronal progenitors, a dearth of calbindin-positive mature neurons and (often) constitutively increased neurogenesis. Although these models provide a link between cellular dysfunction and behavioral alteration, limited translational validity exists linking the iDG to human pathophysiology. In this study, we report the initial identification of an iDG-like phenotype in the hippocampi of human SCZ/BPD patients. These findings suggest a new motif for the etiology of these diseases and link an emerging class of mouse models to the human disease condition.

Translational Psychiatry (2012) 2, e135; doi:10.1038/tp.2012.56; published online 10 July 2012

\section{Introduction}

Difficulties in understanding the etiology and mechanisms of schizophrenia (SCZ), and bipolar disorder (BPD) have been long-standing obstacles in the development of new therapeutics. This difficulty is exacerbated by the fact that the root causes of SCZ/BPD are unknown. With limited clinical investigation possible within the human CNS, rodent models are an obvious alternative for studying these diseases. Although many rodent models for SCZ/BPD exist, few offer translational validity, and little consensus exists on which models are best at recapitulating the disease symptoms and pathologies. ${ }^{1}$

Although SCZ and BPD have distinct clinical criteria for diagnosis, these diseases display significant overlap in symptoms. $^{2}$ In particular, SCZ and BPD patients share cognitive deficits, many of which have been linked to altered or impaired hippocampal function. ${ }^{3}$ As such, it is widely believed that molecular and cellular changes to this region of the brain contribute to SCZ/BPD etiology. Following this line of reasoning, we sought to identify a conserved, recurring motif in the hippocampus of rodent models based on the identification of comparable cognitive deficits to those appreciated in SCZ/BPD patients (described in ref. 4). We used a large-scale comprehensive behavioral battery on more than 100 mutant rodent strains to identify mice with behavioral traits corresponding to SCZ/BPD. From these experiments, we were able to identify four independent strains whose behavioral profile warranted pathophysiological investigation of the brain. Rigorous study of the hippocampus of these mice revealed a common, conserved motif within the hippocampus of each strain, now termed an 'immature dentate gyrus' (iDG). Animals possessing an iDG are defined by increased expression of immature neuronal markers (including doublecortin and calretinin) and reduced levels of mature neuronal markers, most notably calbindin. A secondary feature of IDG mice is elevated neurogenesis, which is present in most iDG mouse lines.

To date, identified iDG strains include the alpha-calcium-/ calmodulin-dependent protein kinase II heterozygous knockout $($ CaMKIl $\alpha-h K O),{ }^{5}$ calcineurin conditional knockout, ${ }^{6,7}$ SNAP-25 knock-in ${ }^{8}$ and schnurri-2 knockout mice. ${ }^{9}$ These strains display the core features of iDG (elevated calretinin and reduced calbindin expression) and elevated proliferation within the subgranular zone (SNAP-25 knock-in mice being a notable exception). Interestingly, iDG mice frequently display several positive and negative behavioral traits that are reminiscent of traits expressed in these psychiatric disorders (including hyperactivity, deficits in social interaction, nest building and working memory).

Although the discovery of an iDG in these strains strongly suggests a link between IDG and the distinct behavioral traits of SCZ/BPD, the translational validity of this class of mouse models remains uncertain, as an iDG-like phenotype has not yet been identified in human patients. To explore this possibility, we examined human cohorts for SCZ and BPD for the molecular hallmarks of the iDG. Human SCZ and BPD patients displayed significantly elevated calretinin expression in the dentate gyrus and trending declines in calbindin expression (in BPD patients) when compared with control subjects and patients suffering from major depression.

${ }^{1}$ CNS, Astellas Research Institute of America LLC, Skokie, IL, USA; ${ }^{2}$ Master of Biotechnology Program, Northwestern University, Evanston, IL, USA; ${ }^{3}$ Stanley Brain Research Laboratory, Stanley Medical Research Institute, Rockville, MD, USA and ${ }^{4}$ Institute for Comprehensive Medical Science, Fujita Health University, Toyoake, Japan

Correspondence: Dr NM Walton, CNS, Astellas Research Institute of America LLC, 8045 Lamon Ave, Skokie, IL 60077, USA.

E-mail: noah.walton@us.astellas.com

Keywords: bipolar disorder; cognition; hippocampus; model; neurogenesis; schizophrenia

Received 25 April 2012; revised 30 May 2012; accepted 31 May 2012 
Calretinin overexpression in both SCZ and BPD patients was closely associated with a psychosis diagnosis and suicide risk. These findings reveal a novel hippocampal alteration in human SCZ/BPD patients and suggest strong validity from this class of mouse models.

\section{Materials and methods}

Animal handling. Young adult (P90) C57BL/6 mice $(n=6)$ were used for all experiments. Alpha-calcium-/calmodulindependent protein kinase II heterozygous knockout mice (CaMKIl $\alpha-\mathrm{hKO}$ ) were generated and described previously. ${ }^{10}$ Mice were singly housed following weaning and maintained under standard housing conditions. All protocols involving animals were developed and conducted in accordance with institutional IACUC protocols. Animals were deeply anesthetized with ketamine-xylazine (80 and $5 \mathrm{mg} / \mathrm{kg}$, respectively) and sacrificed before tissue collection. Cardiac perfusion was performed using $4 \%$ ice-cold paraformaldehyde. Brains were removed and placed in $4 \%$ paraformaldehyde overnight and then transferred to $30 \%$ sucrose solution. For short-term BrdU incorporation studies, $100 \mathrm{mg} / \mathrm{kg}$ (5'-bromodeoxyuridine (BrdU); Sigma, St Louis, MO, USA) was injected and animals were sacrificed 3 days later. Longterm BrdU incorporation was measured 28 days following two injections of $100 \mathrm{mg} / \mathrm{kg} \mathrm{BrdU}$ given $12 \mathrm{~h}$ apart.

Immunohistochemistry. For rodent tissue, serial $20-\mu \mathrm{m}$ coronal sections were cut on a Leica CM 3050 S freezing microtome (Leica Microsystems; Wetzlar, Germany) and stored at $4{ }^{\circ} \mathrm{C}$ in cryoprotectant $(0.1 \mathrm{M}$ phosphate buffer containing: sucrose $(30 \% \mathrm{w} / \mathrm{v})$, ethylene glycol $(30 \% \mathrm{v} / \mathrm{v})$, polyvinyl-pyrrolidone (PVP-40; $1 \% \mathrm{w} / \mathrm{v}$ ). For analysis, sections were removed from cryoprotectant, washed twice in phosphate-buffered saline (PBS, $\mathrm{pH} 7.4$ ) and pretreated with PBS containing $0.3 \%$ Triton $\mathrm{X}-100$. Tissues were blocked for $1 \mathrm{~h}$ at room temperature in PBS containing $0.05 \%$ Triton X-100, 5\% normal donkey serum and 5\% normal goat serum. Primary antibodies included: BrdU (rat monoclonal, 1:500, Abcam; Cambridge, MA, USA), Calbindin (rabbit polyclonal, 1:1000; Swant, Bellinoza, Switzerland) and Calretinin (rabbit polyclonal, 1:1000; Swant). Primary antibodies were applied overnight on an orbital shaker at $4{ }^{\circ} \mathrm{C}$. Sections were washed three times in PBS, and then incubated with secondary antibodies on an orbital shaker for $1 \mathrm{~h}$ at room temperature. Secondary antibodies included Cy2-conjugated goat anti-rat and donkey anti-rabbit (1:600; Jackson Immunoresearch; West Grove, PA, USA). Primary and secondary antibodies were diluted in PBS containing $5 \%$ normal donkey serum. For BrdU imaging, cells were pretreated with sodium chloride/sodium citrate -formamide $\left(1: 1,37^{\circ} \mathrm{C}, 2 \mathrm{~h}\right)$, washed three times for $10 \mathrm{~min}$ in sodium chloride/sodium citrate, incubated in $2 \mathrm{~N} \mathrm{HCl}\left(37^{\circ} \mathrm{C}, 30 \mathrm{~min}\right)$ and washed with $0.1 \mathrm{~m}$ borate buffer $\left(25^{\circ} \mathrm{C}, 10 \mathrm{~min}\right)$. Labeled samples were mounted in Vectastain anti-fade media containing DAPI (Vector Labs, Burlingame, CA, USA) and visualized on a BZ-9000 microscope (Keyence; Chicago, IL, USA). For unbiased stereology, every third hippocampal section was selected for analysis. BrdU-positive cells were manually counted and expressed as cells or section. Golgi staining was performed on $200 \mu \mathrm{m}$ coronal sections from 90-day-old wild-type and CaMKIl $\alpha$-hKO mice using the Rapid Golgistain kit (FD Neurotechnologies, Columbia, MD, USA).

Sequential, coronal sections through mid-hippocampus of the human brain were obtained from Stanley Medical Research Institute neuropathology consortium. Four groups were analyzed: SCZ, BPD, major depressive disorder and unaffected controls (CON) ( $n=15$ in each group). Paraffin sections were $10 \mu \mathrm{m}$ thick and contained the dentate gyrus (DG) (except for one BPD sample, which was not included in this analysis). Tissues were processed for fluorescencebased immunohistochemistry according to the standard procedures. Antigens examined included proliferating cell nuclear antigen (PCNA; mouse monoclonal, 1:600; Sigma), calretinin and calbindin (both from Swant). Images were counterstained with DAPI. Matched-exposure, whole DG captures were taken at $\times 20$ with a Keyence BZ-9000 microscope and reconstructed using proprietary software. The DG granular cell layer area was defined by classic DAPI expression, and did not include hilar expression. Quantification of immunohistochemistry (IHC) intensity for calbindin and calretinin within the DG was performed using ImageJ (National Institutes of Health, Bethesda, MD, USA) and calculated in terms of expression per unit area. PCNApositive cells in the granular cell layer were counted manually and recorded as a fraction of total (DAPI-positive) DG cell number. Values for calbindin, calretinin and PCNA expression are expressed as fraction of control group. Statistical analysis was conducted using PRISM (Graphpad software, La Jolla, CA, USA) and XLSTAT Pro (Addinsoft, New York, NY, USA). One-way analysis of variance (ANOVA) followed by post hoc analysis using Dunnett's comparison test was used for initial assessment of IHC intensity among diagnostic groups. Planned comparisons between control and diagnostic groups were also performed using two-tailed unpaired $t$-test. Effects of clinical variables were assessed by two-tailed unpaired $t$-test (suicide and psychosis), Pearson's correlation coefficients (onset, duration and lifetime quantity of fluphenazine or equivalent) and Spearman's correlation coefficients (severity of alcohol and substance abuse). Pearson's correlation coefficients were used to assess the effects of potential confounding demographic factors including age, postmortem interval, brain $\mathrm{pH}$, brain weight and storage days. Effects of nominal demographic variables, gender and brain hemisphere, were assessed by two-tailed unpaired $t$-test. If significant correlations with demographic variables were observed, analysis of covariance (ANCOVA) was conducted with significantly correlated factors as covariates to assess their influence on effects of diagnosis.

\section{Results}

To confirm the presence of iDG in rodents, we characterized a representative mouse model, in this case the CaMKIl $\alpha$-hKO mouse strain. CaMKIl $\alpha$-hKO mice are a well-established model, and are previously described to possess a highly conserved iDG phenotype at the behavioral and molecular levels. ${ }^{5}$ CaMKIl $\alpha$-hKO mice display deficits in many mouse behaviors identified as cognates of positive, negative and 

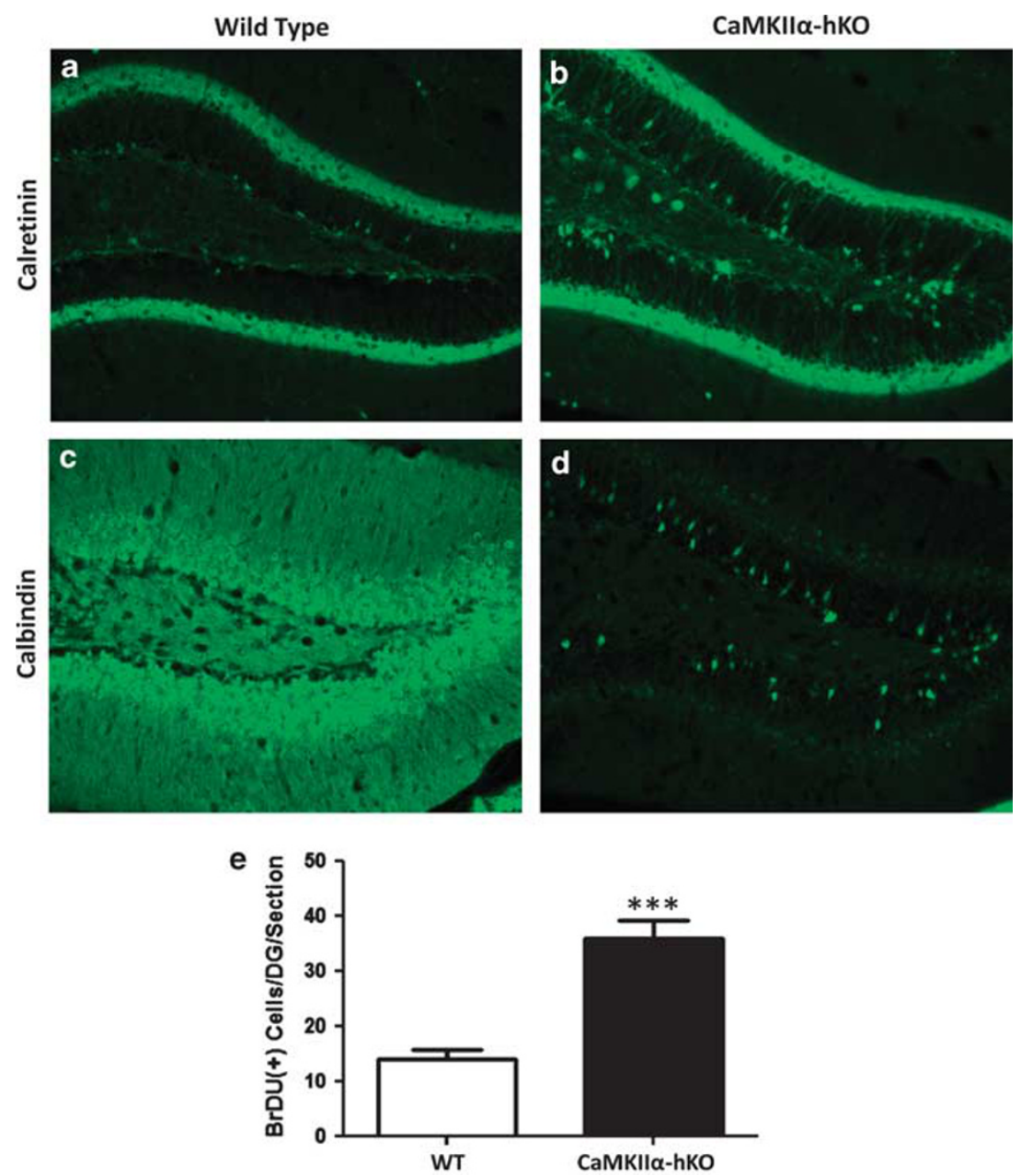

Figure 1 CaMKIl $\alpha$-hKO mice, a representative mouse model of immature dentate gyrus' (iDG), possess a perpetually immature hippocampal formation. Expression of early neuronal markers is elevated in mutant mice (calretinin shownin $\mathbf{a}, \mathbf{b}$ ), which display a deficit in the mature neuronal marker calbindin (c, d). Neurogenesis is substantially increased in the dentate gyrus of mutant iDG mice (e). Scale bar $=100 \mu \mathrm{m} .{ }^{* \star} \mathrm{P}<0.001$.

cognitive features of SCZ/BPD, including hyperactivity, reduced social interaction, impaired novel exploration in $\mathrm{Y}$-maze, trace and contextual fear conditioning memory (Supplementary Figure 1). Comparative analysis of the hippocampi of CaMKIl $\alpha$-hKO to controls confirmed the iDG molecular phenotype: Calretinin expression was significantly increased in CaMKIl $\alpha$-hKO mice (Figures $1 \mathrm{a}$ and $\mathrm{b}$; Doublecortin expression was also significantly increased, data not shown), whereas the mature neuronal marker calbindin was significantly reduced in mutant mice (Figures 1c and d). Acute BrdU labeling of CaMKIl $\alpha$-hKO mice revealed a significant increase in proliferation within the subgranular zone (Figure 1e).

Based on the behavioral similarities (particularly the hippocampus-associated cognitive deficits) between iDG mice and human SCZ/BPD patients, we hypothesized that human patients may possess cellular/molecular correlates of iDG. Sequential mid-hippocampal coronal sections of human brain were obtained from the Stanley Medical Research Institute neuropathology consortium, which contained four groups: SCZ, BPD, major depressive disorder and CON $(n=15$ in each group, Table 1). Quantitative immunohistochemistry was performed with antibodies labeling the immature neuronal marker calretinin, the mature neuronal marker calbindin and the proliferation-associated marker PCNA.

Calretinin was significantly upregulated in the DG of both SCZ and BPD patients but not in patients suffering from depression (Figure 2a; ANOVA, $\mathrm{F}(3,55)=6.42, P=0.0008$; CON vs SCZ, $P<0.05$; CON vs BPD, $P<0.01)$. Calretinin was highly expressed in the DG and, to a lesser extent, $C A 1$ in SCZ/BPD patients compared with controls (Figures $2 d-f$ ). By comparing calretinin expression with clinical data for patients, we found that increased calretinin expression positively correlated with suicide death (Figure $2 \mathrm{~g} ; t=2.56$, df $=57$, $P=0.013$ ), psychosis (Figure $2 \mathrm{~h} ; t=3.83, \quad \mathrm{df}=57$, $P=0.0003$ ) and duration of disease (Figure 2i; $r=0.36$, $P=0.017)$, and negatively correlated with age of onset (Figure 2j; $r=-0.46, P=0.0017)$. No correlations were found with other clinical data, namely lifetime quantity of neuroleptics treatment or severity of alcohol and substance abuse. No correlations were found between calretinin expression levels and potential demographic confounding variables except for postmortem interval $(r=0.26, P=0.047)$ and storage days $(r=0.39, P=0.0021)$. When we tested these two confounding factors as covariates in ANCOVA, 
Table 1 Demographic and clinical data of subjects of the Stanley foundation neuropathology consortium used in this study

\begin{tabular}{|c|c|c|c|c|}
\hline & $\begin{array}{c}\text { Control, } \\
\mathrm{N}=15\end{array}$ & $\begin{array}{l}\text { Schizophrenia, } \\
\qquad N=15\end{array}$ & $\begin{array}{l}\text { Bipolar disorder, } \\
\qquad N=15\end{array}$ & $\begin{array}{c}\text { Depression, } \\
\mathrm{N}=15\end{array}$ \\
\hline $\begin{array}{l}\text { Gender (M/F) } \\
\text { Age (year) } \\
\text { Post mortem interval (hour) } \\
\text { Brain pH } \\
\text { Brain hemisphere (R/L) } \\
\text { Brain weight (g) } \\
\text { Storage days } \\
\text { Age of onset (year) } \\
\text { Duration of illness (year) } \\
\text { History of psychosis } \\
\text { Suicide death } \\
\text { Lifetime fluphenazine equivalent (mg) }\end{array}$ & $\begin{array}{c}9 / 6 \\
48.1 \pm 10.7 \\
23.7 \pm 9.9 \\
6.27 \pm 0.24 \\
7 / 8 \\
1501 \pm 164 \\
338 \pm 234 \\
- \\
- \\
0 \\
0 \\
-\end{array}$ & $\begin{array}{c}9 / 6 \\
44.5 \pm 13.1 \\
33.7 \pm 14.6 \\
6.16 \pm 0.26 \\
6 / 9 \\
1472 \pm 108 \\
621 \pm 233 \\
23.2 \pm 8.0 \\
21.3 \pm 11.4 \\
15 \\
4 \\
52270 \pm 62060\end{array}$ & $\begin{array}{c}9 / 6 \\
42.3 \pm 11.7 \\
32.5 \pm 16.1 \\
6.18 \pm 0.23 \\
8 / 7 \\
1441 \pm 172 \\
621 \pm 172 \\
21.5 \pm 8.3 \\
20.1 \pm 9.7 \\
11 \\
9 \\
20830 \pm 24020\end{array}$ & $\begin{array}{c}9 / 6 \\
46.5 \pm 9.3 \\
27.5 \pm 10.7 \\
6.18 \pm 0.21 \\
6 / 9 \\
1462 \pm 142 \\
434 \pm 290 \\
33.9 \pm 13.3 \\
12.7 \pm 11.1 \\
0 \\
7 \\
-\end{array}$ \\
\hline $\begin{array}{l}\text { Drug use } \\
\text { Little/none } \\
\text { Social } \\
\text { Moderate use, past } \\
\text { Moderate use, present } \\
\text { Heavy use, past } \\
\text { Heavy use, present }\end{array}$ & $\begin{array}{c}14 \\
1\end{array}$ & $\begin{array}{l}10 \\
1 \\
1 \\
2 \\
1\end{array}$ & $\begin{array}{l}5 \\
1 \\
2\end{array}$ & 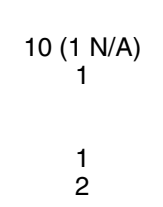 \\
\hline $\begin{array}{l}\text { Alcohol use } \\
\text { Little/none } \\
\text { Social (1-2 drinks/day) } \\
\text { Moderate use, past } \\
\text { Moderate use, present } \\
\text { Heavy use, past } \\
\text { Heavy use, present }\end{array}$ & $\begin{array}{l}5 \\
6 \\
2 \\
2 \\
0 \\
0\end{array}$ & $\begin{array}{l}5 \\
3 \\
2 \\
2 \\
2 \\
1\end{array}$ & $\begin{array}{c}1(1 \mathrm{~N} / \mathrm{A}) \\
4 \\
1 \\
2 \\
3 \\
3\end{array}$ & $\begin{array}{l}5 \\
5 \\
0 \\
1 \\
1 \\
3\end{array}$ \\
\hline
\end{tabular}

Abbreviations: M/F, male/female; R/L, right or left; N/A, not available.

differences in calretinin expression among four diagnosis groups were still statistically significant (ANCOVA, F(3, $53)=2.98, P=0.04$ ). More importantly, these confounding factors have lesser impacts compared with diagnosis (ANCOVA; postmortem interval, $P=0.25$, storage days, $P=0.11$, diagnosis, $P=0.04$ ).

ANOVA analysis of calbindin expression did not show statistical differences between groups (Figure 2b; ANOVA, $\mathrm{F}(3,55)=1.97, P=0.13)$. However, we found trending decreases in calbindin expression in BPD patients (Figure 2b; two-tailed unpaired $t$-test; CON vs BPD, $t=1.78$, $\mathrm{df}=27, P=0.086)$. We found no associations between calbindin expression levels and clinical data for patients. No correlations were found between calbindin expression levels and potential demographic confounding variables except for storage days $(r=-0.29, P=0.025)$. When we tested storage days as a covariate in ANCOVA, differences in calbindin expression among four diagnosis groups did not reach statistically significant levels (ANCOVA, $F(3,54)=1.89$, $P=0.14)$. Although we did not detect decreased calbindin expression, a larger gene expression study in the dentate gyrus detected a significant decrease in calbindin messenger expression in SCZ DG, ${ }^{11}$ as well as protein-level alterations in other regions of SCZ patients. ${ }^{12,13}$

No difference was detected in PCNA expression among four diagnostic groups (Figure 2c; ANOVA, $F(3,55)=0.27$, $P=0.84)$. We found no associations between PCNA expression levels and clinical data for patients. No correlations were found between PCNA expression levels and potential demographic confounding variables except for a strong positive correlation with storage days $(r=0.43, P=0.0007)$. When we tested storage days as a covariate in ANCOVA, differences in PCNA expression among four diagnosis groups did not reach statistically significant levels (ANCOVA, $F(3,54)=2.22$, $P=0.096)$. Moreover, the fact that storage days have a strong impact compared with diagnosis (ANCOVA; storage days, $P<0.0001$, diagnosis, $P=0.096$ ) may represent the unreliable nature of PCNA signal detected in this study.

\section{Discussion}

We have described the identification of an iDG-like phenotype in human patients suffering from SCZ/BD. The iDG was previously identified in a growing class of rodent models displaying conserved traits of psychiatric disease. Despite originating from disparate sources of targeted mutations, iDG mutant mice are defined by two traits: behaviors reminiscent of SCZ/BPD individuals (notably strong cognitive deficits) and pathophysiological alteration of the dentate gyrus, specifically the temporally inappropriate exhibition of cellular and molecular hallmarks of immaturity well into adulthood.

On a cellular/molecular level, the core features of iDG are an increased level of immature neuronal markers (including calretinin and doublecortin) and a lack of the mature neuronal marker calbindin. A secondary feature is enhanced hippocampal proliferation (presumably of subgranular neural stem cells (NSCs)), which has been appreciated in the majority of iDG mouse models to date. Although the iDG is an increasingly well-characterized model for psychiatric diseases in rodents, the hallmarks of this condition were previously uncharacterized in human patients. Using quantitative IHC, we appreciated increased calretinin expression in BPD/SCZ patients, along with trending decreases in calbindin expression in BPD patients. The recapitulation of the core features of this class of mouse disease models in human patients demonstrates the presence 

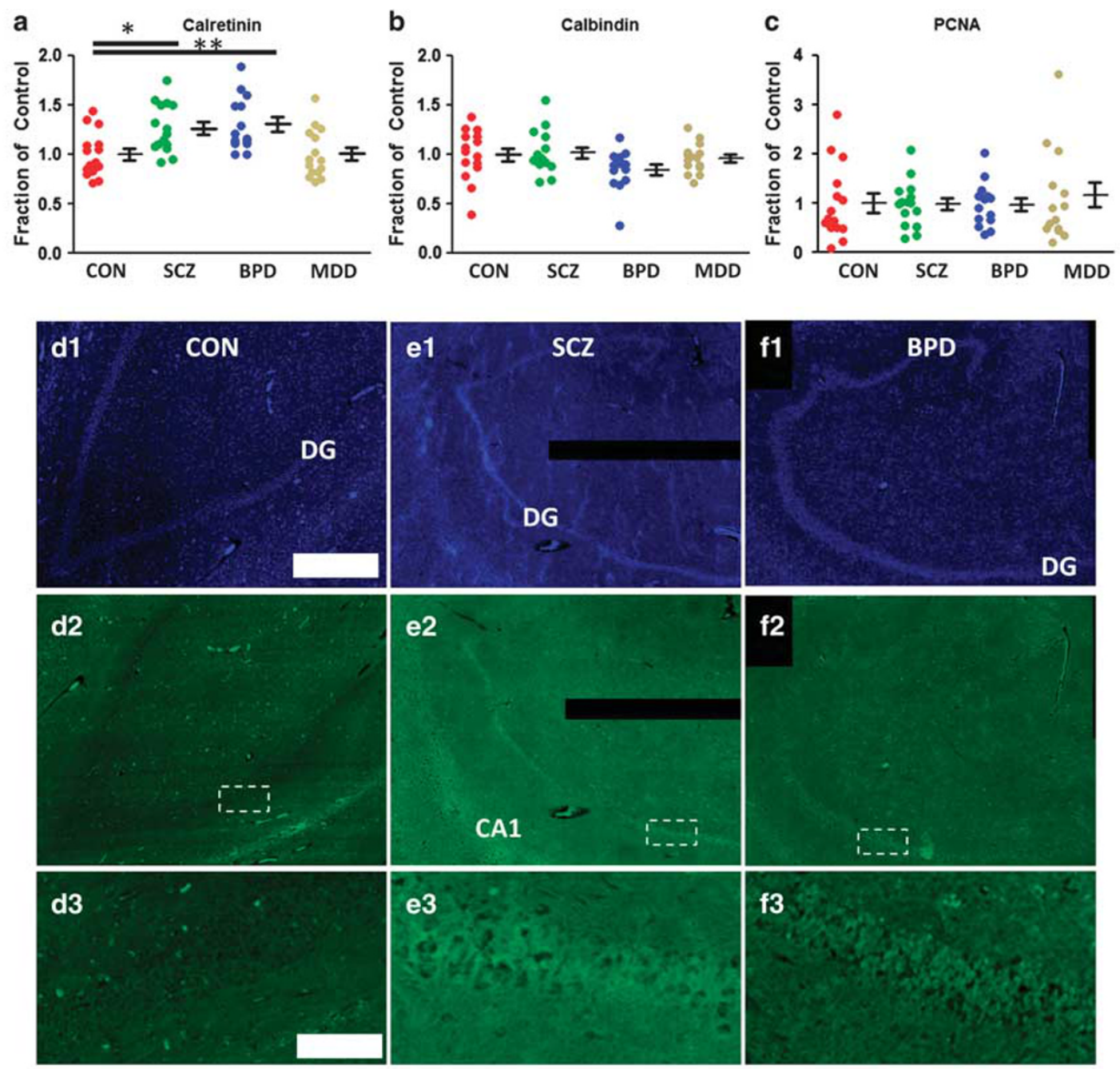

g

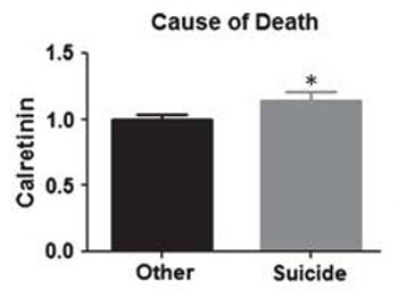

h

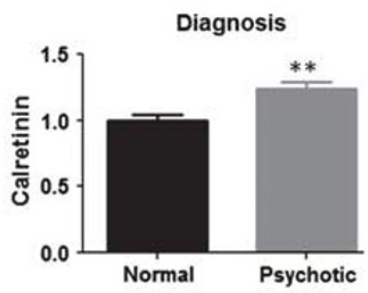

i

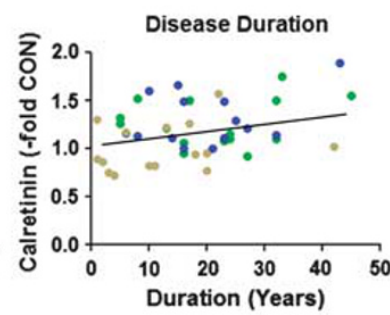

j

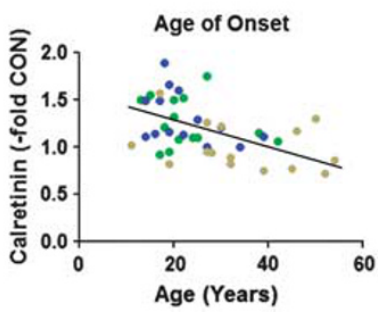

Figure 2 Identification of immature dentate gyrus' (iDG) in a subset of schizophrenia/bipolar disorder (SCZ/BPD) patients. Expression of (a) calretinin, (b) calbindin and (c) PCNA ratios within the dentate gyrus (DG) of SCZ, BPD and major depressive disorder (MDD) cohorts. Calretinin was consistently overexpressed in DG neurons in SZ/BPD patients vscontrol (d2-f2; boxed areas magnified in d3-f3; DAPI d1-f1). Elevated calretinin expression is positively associated with (g) suicide death, (h) psychosis diagnosis and (i) disease duration and (j)negatively associated with age at disease onset. (i-j) SCZ = green, BPD = blue, MDD = brown. Scale bars: $1 \mathrm{~mm}(\mathbf{d}-\mathbf{f} 1,2) ; 100 \mu \mathrm{m}(\mathbf{d}-\mathbf{f} 3$ ). $P$ values: ${ }^{*}<0.05,{ }^{* *}<0.01$.

of an iDG-like condition in human patients. The elevated expression of calretinin is particularly remarkable, as the expression of other early neuronal markers like doublecortin are reported to decrease with age. ${ }^{14}$ Additionally, these findings provide a novel link between emerging mouse models and human disease condition, providing a new tool in the basic science and drug discovery surrounding SCZ and BPD.
The relationship between the iDG phenotype and the SCZ/ BPD-like behavioral symptoms remains largely unexplored. One clue may lie in enhanced neurogenesis' contribution to hippocampus-dependent behaviors. One popular theory suggests that cognitive deficits in SCZ patients may be related to deficits in temporal encoding of new memories, a popularly theorized role of adult neurogenesis. ${ }^{15}$ Temporal 
encoding processes, in turn, may be affected by alterations in the critical period of development, which are essential for neuronal development. ${ }^{16}$ CaMKIl $\alpha$-hKO mice consistently exhibit deficits in the generation of mature morphologies (particularly granule cell neurons, as evidenced by Golgi staining, Supplementary Figure $2 a-d$ ), suggesting possibly that progeny arising from neurogenesis do not fully mature through this critical period. Whether this is the case in humans is not yet clear.

The reasons for the increased presence of immature cells within the dentate gyrus remain largely unknown. It is unclear whether the large number of calretinin/doublecortin-positive cells observed are a result of the loss of mature cells (that is, compensatory elevated neurogenesis) or whether they are accumulated as a result of a defect in the normal maturational pathway. Evidence exists to support each of these possibilities (which are themselves nonexclusive). We were able to detect nonsignificant decreases in calbindin expression in BPD patients, and other studies have detected a decrease in calbindin in the dentate gyrus of SCZ patients. ${ }^{11}$ The iDG mice do not display significantly lower rates of long-term progeny survival than WT mice (Supplementary Figure 2e); However, multiple studies have detected alterations in interleukins and other markers of oxidative stress, suggesting that cell turnover may occur in SCZ patients. ${ }^{17}$ Undoubtedly, the biological underpinnings of this phenomenon will be the subject of future work.

Elevated neurogenesis has been defined as a secondary core feature of iDG pathology, and the contributions of postnatally generated neurons are increasingly implicated in the formation and retention of new memories in rodent models. ${ }^{16}$ In human patients, the constitutive elevation of neurogenic frequency may have an effect on hippocampal function, as well as effects on the long-term maintenance of populations of NSCs. Using a similar patient set, Reif et al. ${ }^{18}$ claim a decrease in neurogenesis in SCZ patients. However, the authors of that study excluded several patients with levels of neurogenesis greater than fivefold basal levels, which may represent a subset of SCZ/BPD patients displaying hyperactive neurogenesis. Furthermore, Reif et al. rely on $\mathrm{Ki}-67$ labeling of dividing cells, an antigen which subsequent work suggests declines in early- to mid-adulthood. ${ }^{14}$ The latter study suggests PCNA as an excellent longitudinal marker of cell division within the central nervous system (CNS). Using PCNA as an antigen, we were unable to detect increased proliferation in the dentate gyrus. Emerging studies on the longevity of NSCs may resolve this discrepancy: New studies suggest that NSCs in the hippocampus are progressively depleted with age, ${ }^{19}$ suggesting pools of NSCs may exhaust more rapidly (and variably) with elevated levels of neurogenesis.

The finding of an iDG-like condition in human SCZ/BPD patients presents a tantalizing possibility of a new mouse model offering the prospect of reliable translational validity. Although other measures of predictive validity (for example, the response of iDG mouse models to conventional anti- psychotics) are yet unknown, establishing reliable models for diseases with unknown etiologies is an important early step in the elucidation of basic disease mechanisms and the development of novel therapeutics.

\section{Conflict of interest}

The authors declare no conflict of interest.

1. Young JW, Zhou X, Geyer MA. Animal models of schizophrenia. Curr Top Behav Neurosci 2010; 4: 391-433.

2. Walker J, Curtis V, Shaw P, Murray RM. Schizophrenia and bipolar disorder are distinguished mainly by differences in neurodevelopment. Neurotox Res 2002; 4: 427-436.

3. Schretlen DJ, Cascella NG, Meyer SM, Kingery LR, Testa SM, Munro CA et al. Neuropsychological functioning in bipolar disorder and schizophrenia. Biol Psychiatry 2007; 62: 179-186.

4. Powell CM, Miyakawa T. Schizophrenia-relevant behavioral testing in rodent models: a uniquely human disorder? Biol Psychiatry 2006; 59: 1198-1207.

5. Yamasaki N, Maekawa M, Kobayashi K, Kajii Y, Maeda J, Soma M et al. Alpha-CaMKII deficiency causes immature dentate gyrus, a novel candidate endophenotype of psychiatric disorders. Mol Brain 2008; 1: 6.

6. Hagihara H, Nakamura HK, Toyama K, Graeff IA, Crabtree GR, Miyakawa T. Paper presented at the 41st Annual Meeting of the Society for Neuroscience, Washington, DC; 12 to 16 November 2011.

7. Miyakawa T, Leiter LM, Gerber DJ, Gainetdinov RR, Sotnikova TD, Zeng $\mathrm{H}$ et al. Conditional calcineurin knockout mice exhibit multiple abnormal behaviors related to schizophrenia. Proc Natl Acad Sci USA 2003; 100: 8987-8992.

8. Ohira K, Toyama K, Nakamura HK, Shoji H, Kayaoka M, Takahashi M et al. Paper presented at the 41st Annual Meeting of the Society for Neuroscience, Washington, DC; 12 to 16 November 2011.

9. Takao K, Hagihara H, Ohira K, Toyama K, Takagi T, Ishii S et al. Paper presented at the 41st Annual Meeting of the Society for Neuroscience, Washington, DC; 12 to 16 November 2011.

10. Silva AJ, Stevens CF, Tonegawa S, Wang Y. Deficient hippocampal long-term potentiation in alpha-calcium-calmodulin kinase II mutant mice. Science 1992; 257: 201-206.

11. Altar CA, Jurata LW, Charles V, Lemire A, Liu P, Bukhman Y et al. Deficient hippocampal neuron expression of proteasome, ubiquitin, and mitochondrial genes in multiple schizophrenia cohorts. Biol Psychiatry 2005; 58: 85-96.

12. Cotter D, Landau S, Beasley C, Stevenson R, Chana G, MacMillan L et al. The density and spatial distribution of GABAergic neurons, labelled using calcium binding proteins, in the anterior cingulate cortex in major depressive disorder, bipolar disorder, and schizophrenia. Biol Psychiatry 2002; 51: 377-386

13. Reynolds GP, Zhang ZJ, Beasley CL. Neurochemical correlates of cortical GABAergic deficits in schizophrenia: selective losses of calcium binding protein immunoreactivity. Brain Res Bull 2001; 55: 579-584.

14. Knoth R, Singec I, Ditter M, Pantazis G, Capetian P, Meyer RP et al. Murine features of neurogenesis in the human hippocampus across the lifespan from 0 to 100 years. PLoS One 2010; 5: e8809.

15. Reif A, Schmitt A, Fritzen S, Lesch KP. Neurogenesis and schizophrenia: dividing neurons in a divided mind? Eur Arch Psychiatry Clin Neurosci 2007; 257: 290-299.

16. Aasebo IE, Blankvoort S, Tashiro A. Critical maturational period of new neurons in adult dentate gyrus for their involvement in memory formation. Eur $J$ Neurosci 2011; 33: 1094-1100.

17. Wood SJ, Yucel M, Pantelis C, Berk M. Neurobiology of schizophrenia spectrum disorders: the role of oxidative stress. Ann Acad Med Singapore 2009; 38: 396-6.

18. Reif A, Fritzen S, Finger M, Strobel A, Lauer M, Schmitt $A$ et al. Neural stem cell proliferation is decreased in schizophrenia, but not in depression. Mol Psychiatry 2006; 11: 514-522.

19. Encinas JM, Michurina TV, Peunova N, Park JH, Tordo J, Peterson DA et al. Divisioncoupled astrocytic differentiation and age-related depletion of neural stem cells in the adult hippocampus. Cell Stem Cell 2011; 8: 566-579.
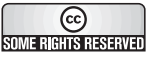

Translational Psychiatry is an open-access journal published by Nature Publishing Group. This work is licensed under the Creative Commons Attribution-Noncommercial-Share Alike 3.0 Unported License. To view a copy of this license, visit http:// creativecommons.org/licenses/by-nc-sa/3.0/

Supplementary Information accompanies the paper on the Translational Psychiatry website (http://www.nature.com/tp) 